\title{
ANÁLISE DE INDICADORES DE DESEMPENHO DA ILUMINAÇÃO ARTIFICIAL EM AMBIENTES RESIDENCIAIS
}

\section{ANALYSIS OF ARTIFICIAL LIGHTING PERFORMACE INDICATORS FOR RESIDENTIAL BUILDINGS}

\author{
Júlia Silva de Moraes 1 \\ Universidade Federal de Alagoas, Maceió, Alagoas, Brasil, arq.juliademoraes@gmail.com \\ Adrián Muros Alcojor ${ }^{2}$ \\ Universidad Politécnica de Cataluña, Barcelona, Catalunha, Espanha, adrian.muros@upc.edu \\ Leonardo Salazar Bittencourt ${ }^{3}$ \\ Universidade Federal de Alagoas, Maceió, Alagoas, Brasil, 1sb54@hotmail.com
}

\section{Resumo}

A utilização de indicadores para a avaliação de desempenho dos sistemas artificiais de iluminação no ambiente construído vem sendo discutida em vários países. No entanto, poucas são as ferramentas que permitem conciliar aspectos quantitativos e qualitativos da iluminação com eficiência energética durante a elaboração de projetos de iluminação artificial. Este trabalho compara os indicadores brasileiros com os principais indicadores internacionais, entendidos como valores referenciais que auxiliam o projetista em relação ao desempenho de projetos de iluminação. Para tal, foi realizada uma pesquisa documental nas normas e códigos dos indicadores relacionados ao conforto visual e à eficiência energética da iluminação em ambientes residenciais, bem como uma análise comparativa das semelhanças e diferenças mais significativas dentre os indicadores existentes. Como resultado, observou-se que os indicadores nacionais são genéricos, desconsiderando as especificidades das atividades exercidas em ambientes residenciais. A pesquisa documental demonstrou a carência de indicadores que melhor expressem o desempenho da iluminação artificial em ambientes residenciais. Tal situação tem levado os projetistas a recorrer aos indicadores existentes nos manuais e regulamentos referentes às tipologias não residenciais como parâmetro de desempenho a ser alcançado. A relevância do presente trabalho consiste na identificação de lacunas importantes existentes nas normas e códigos, contribuindo para o aperfeiçoamento das referidas normas nacionais e seus reflexos na atividade projetual da área em estudo.

Palavras-chave: Indicadores de desempenho. Iluminação artificial. Normas técnicas.

\begin{abstract}
The use of indicators for the performance evaluation of artificial lighting systems in the built environment has been discussed in several countries. However, there are few tools to reconcile quantitative and qualitative aspects of energy-efficient lighting during the design of artificial lighting projects. This work compares the Brazilian indicators with the leading international indicators, understood as reference values that help the designer concerning the performance of lighting projects. Documentary research was carried out on the standards and codes of indicators related to visual comfort and energy efficiency of lighting in residential environments, as well as a comparative analysis of similarities and significant differences among them. As a result, it was observed that the national indicators are generic, disregarding specificities of activities carried out in residential environments. The study has demonstrated a lack of indicators that best express the performance of artificial lighting in residential environments. This situation has led the designers to use existing indicators in the manuals and regulations regarding non-residential typologies as a parameter of performance to be achieved. The relevance of this work is the identification of essential gaps in standards and codes, contributing to the improvement of these national standards and their impact on the project activity of the area under study.
\end{abstract}

Keywords: Indicators. Artificial lighting. Building Regulations.

How to cite this article:

MORAES, Júlia Silva de; MUROS ALCOJOR, Adrián; BITTENCOURT, Leonardo Salazar. Análise de indicadores de desempenho da iluminação artificial em ambientes residenciais. PARC Pesquisa em Arquitetura e Construção, Campinas, SP, v. 9, n. 1, p. 35-46, mar. 2018. ISSN 19806809. Disponível em: <https://periodicos.sbu.unicamp.br/ojs/index.php/parc/article/view/8650219>. Acesso em: 10 maio 2018. doi:doi:https://doi.org/10.20396/parc.v9i1.8650219. 


\section{Introdução}

O uso de indicadores de desempenho da iluminação artificial aplicados ao projeto de arquitetura vem sendo gradativamente incorporado pelos projetistas. No entanto, poucos permitem conciliar aspectos quantitativos e qualitativos da iluminação nos ambientes habitados.

A eficiência dos sistemas de iluminação artificial pode ser avaliada pelo uso de indicadores de desempenho. Estes indicadores são entendidos como valores referenciais que auxiliam o projetista em relação ao desempenho da iluminação que se deseja obter. "O indicador é uma medida, geralmente quantitativa, usado para ilustrar e comunicar fenômenos complexos de forma simples, incluindo as tendências e os progressos ao longo do tempo". (EEA, 2005. p.7).

$\mathrm{O}$ uso de indicadores geralmente é associado aos benchmarks, processo sistemático de medir o desempenho e comparar com outros considerados como boas práticas. Sendo utilizado como um padrão a ser seguido, "um benchmark é uma linha de base ou ponto de referência, que pode ser utilizado para avaliar o desempenho de coisas do mesmo tipo" (BORGSTEIN; LAMBERTS, 2014, p. 3).

O Comitê de Energia do Conselho Brasileiro de Construção Sustentável (CBCS, 2013) considera os benchmarks como instrumentos eficazes para redução de consumo de energia no ambiente habitado e ressalta a necessidade de indicadores simples e confiáveis de desempenho e de sustentabilidade. Porém, alerta que faltam informações de desempenho de edificações que possam se tornar referenciais para a comparação com as demais construções, considerando a realidade brasileira.

O projeto de iluminação, enquanto processo dinâmico, necessita de um conjunto de informações específicas que melhor expressem o desempenho dos sistemas de iluminação. Ao comparar seu próprio desempenho com o desempenho de referencial estabelecido, os gerentes ou proprietários de um edifício podem rapidamente estimar o seu desempenho (CBCS, 2013).

$\mathrm{Na}$ iluminação de residências brasileiras, que indicadores serão tomados como referenciais para testar e avaliar a pertinência de decisões projetuais? A edificação atenderá a valores mínimos ou se esforçará para superá-los? (GRONDZIK; KWOK, 2013. p.12).

Este trabalho tem como objetivo realizar uma pesquisa documental incluindo normas atuais que abordam a questão dos indicadores de desempenho visual e eficiência da iluminação artificial em ambientes residenciais, de forma a identificar eventuais lacunas do conhecimento nesses documentos. Pretende, ainda, apresentar contribuições para o aperfeiçoamento das normas nacionais e para o projeto dos sistemas de iluminação artificial residencial.

\section{Método}

Para alcançar o objetivo acima delineado, foram examinadas as principais publicações nacionais e internacionais contendo indicadores de desempenho da iluminação artificial em projetos arquitetônicos.

$\mathrm{O}$ processo de pesquisa documental apontou para os manuais, normas e códigos como as principais fontes que abordam essa temática. O procedimento utilizado para o rastreamento das fontes empregadas nesse artigo teve como critério a delimitação de documentos referenciais produzidos por: (i) associações internacionais dedicadas ao estudo e divulgação da iluminação que possuem um comitê internacional com abrangência mundial; (ii) normas e regulamentos de instituições nacionais; (iii) importantes publicações internacionais sobre o tema, oriundas de países com representantes nos comitês internacionais de padronização. Além dessas, foram incluídos normas ou códigos edílicos de países com destaque no cenário internacional, tais como observados na Austrália, Espanha, Estados Unidos - particularmente as normas adotadas pelo estado da Califórnia.

Os indicadores da iluminação foram reunidos em dois grupos: os que apresentam sugestões relativas ao conforto visual e aqueles que contêm recomendações referentes à eficiência energética dos sistemas de iluminação em ambientes.

A reunião documental por grupos auxiliou na análise comparativa das semelhanças e diferenças mais significativas dentre os diversos indicadores examinados, considerando as especificidades das atividades exercidas em ambientes residenciais.

Os indicadores concernentes ao conforto visual expressam condições favoráveis ao desempenho das atividades realizadas em ambientes residenciais. Já a eficiência energética é resultado de dois aspectos complementares. O primeiro refere-se ao desempenho dos equipamentos instalados (lâmpadas, luminárias e complementos) e o segundo depende das características do ambiente construído (forma, dimensões, acabamento das superfícies internas, tipologia das aberturas) e de aspectos comportamentais dos seus usuários.

As grandezas fotométricas apresentadas no Quadro 1 a seguir estão descritas na literatura da iluminação artificial (IES, 2011; SLL, 2009; ABNT, 2013b; ELETROBRAS; UFSC, 2014, 2015). Correspondem às unidades de medida adotadas pelos documentos investigados para o estabelecimento de métricas concernentes ao conforto visual e (ou) dedicadas à obtenção de maior eficiência energética no ambiente habitado. 
Oportunamente, as grandezas fotométricas encontram-se desagregadas nos dois eixos aqui estabelecidos: as métricas em conforto visual e as de eficiência energética dos sistemas de iluminação no ambiente habitado. Essa classificação possibilita identificar com melhor clareza as características de cada grupo e as relações estabelecidas entre os parâmetros analisados.

\begin{tabular}{|c|c|c|c|c|c|}
\hline & Grandezas fotométricas & Símbolo & Unidade & Algoritmo & Definição \\
\hline \multirow{5}{*}{ o } & |lluminância mantida & $\mathrm{EM}_{\mathrm{M}}$ & $\operatorname{lux}$ & $\mathbf{E}_{M}=\frac{\Phi}{A}$ & $\begin{array}{l}\text { Valor abaixo do qual não convém que a } \\
\text { iluminância média da superfície especificada seja } \\
\text { reduzida (ABNT, 2013b). }\end{array}$ \\
\hline & Uniformidade & $U_{0}$ & - & $\mathbf{U}_{\mathbf{o}}=\frac{\mathbf{E}_{\text {min }}}{\mathbf{E}_{\text {med }}}$ & $\begin{array}{l}\text { Razão entre o valor da iluminância mínima (Emin) } \\
\text { e a média (Emed) (ABNT, 2013b). }\end{array}$ \\
\hline & $\begin{array}{l}\text { Índice Limite de } \\
\text { Ofuscamento Unificado }\end{array}$ & UGRL & - & $\mathrm{UGR}_{\mathrm{L}}=8 \log \left(\frac{0,25}{\mathbf{L}_{\mathrm{b}}} \cdot \sum \frac{\mathrm{L}^{2} \cdot \omega}{\mathbf{p}^{2}}\right)$ & $\begin{array}{l}\text { Valor máximo permitido do nivel de ofuscamento } \\
\text { unificado de um projeto para uma instalação de } \\
\text { iluminação - ver detalhamento do índice na } \\
\text { norma (ABNT, 2013b). }\end{array}$ \\
\hline & $\begin{array}{l}\text { Índice Reprodução de Cor } \\
\text { (IRC) }\end{array}$ & $\mathrm{Ra}_{\mathrm{a}}$ & - & - & $\begin{array}{l}\text { Valor percentual que fornece uma indicação } \\
\text { objetiva das propriedades de reprodução de cor } \\
\text { de uma fonte de luz (ABNT, 2013b). }\end{array}$ \\
\hline & $\begin{array}{l}\text { Temperatura de Cor } \\
\text { Correlata }\end{array}$ & $T_{c p}$ & Kelvin $(\mathrm{K})$ & & $\begin{array}{l}\text { Refere-se à cor aparente (cromaticidade da } \\
\text { lâmpada) da luz que ela emite. É dividida em } 3 \\
\text { faixas: Quente - abaixo de } 3.300 K \text {; Intermediária } \\
\text { - entre } 3.300 K \text { e 5.300K; Fria - acima de } 5.300 \mathrm{~K} \\
\text { (ABNT, 2013b). }\end{array}$ \\
\hline \multirow{4}{*}{ 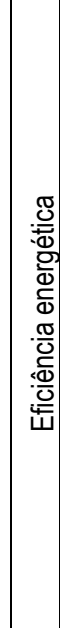 } & Eficiência Luminosa & $\eta$ & $\mathrm{Im} / \mathrm{W}$ & $\eta=\frac{\Phi}{P}$ & $\begin{array}{l}\text { Quociente entre fluxo luminoso emitido ( } \phi) \text {, em } \\
\text { lúmens, pela potência consumida, em Watts. } \\
\text { (ELETROBRAS; UFSC, 2014). }\end{array}$ \\
\hline & $\begin{array}{l}\text { Densidade de Potência da } \\
\text { lluminação }\end{array}$ & DPI & $\mathrm{W} / \mathrm{m}^{2}$ & $D P I=\frac{P}{A}$ & $\begin{array}{l}\text { Razão entre o somatório da potência de } \\
\text { lâmpadas e reatores e a área de um ambiente } \\
\text { (ELETROBRAS; UFSC, 2014). }\end{array}$ \\
\hline & $\begin{array}{l}\text { Densidade de Potência } \\
\text { Limite da lluminação }\end{array}$ & DPIL & $\mathrm{W} / \mathrm{m}^{2}$ & $\operatorname{DPI}_{L}=\frac{P}{A}$ & $\begin{array}{l}\text { Razão entre o somatório da potência de } \\
\text { lâmpadas e reatores e a área de um ambiente, } \\
\text { relação essa considerada eficiente, pois está } \\
\text { diretamente relacionada ao nível de lluminância } \\
\text { necessário nos planos de trabalho, a atividade a } \\
\text { ser executada em cada ambiente ou edifício } \\
\text { (ELETROBRAS; UFSC, 2015). }\end{array}$ \\
\hline & $\begin{array}{l}\text { Densidade de Potência } \\
\text { Relativa }\end{array}$ & $\mathrm{DPl}_{\mathrm{R}}$ & W/m² por 100 lux & $D P I_{R}=\frac{D P I}{E_{M} / 100}$ & $\begin{array}{l}\text { Densidade de potência total instalada para cada } \\
100 \text { lux de lluminância (MINISTÉRIO DE } \\
\text { FOMENTO, } 2013 \text { - adaptado). }\end{array}$ \\
\hline
\end{tabular}

Sendo: $\boldsymbol{\Phi}=$ Fluxo luminoso ( $(\mathrm{Im}) ; \mathrm{A}=$ área $\left(\mathrm{m}^{2}\right) ; \mathrm{L}_{\mathrm{b}}=$ Luminância de fundo $\left(\mathrm{cd} / \mathrm{m}^{2}\right) ; \mathrm{L}=$ Luminância da parte luminosa de cada luminária na direção do olho do observador $\left(\mathrm{cd} / \mathrm{m}^{2}\right) ; \omega=$ ângulo sólido da parte luminosa de cada luminária na direção do olho do observador; P= Potência total instalada em Watts. Fontes: Indicadas no Quadro e adaptadas pelos autores.

\section{Indicadores da iluminação artificial em ambientes residenciais}

Entre as publicações mais utilizadas por profissionais e pesquisadores internacionais estão os manuais das duas mais importantes instituições da área, a Illuminating Engineering Society - IES (2011) e a The Society of Light and Lighting - SLL (2009). As duas instituições possuem um comitê internacional de pesquisadores que atualizam conhecimentos e discutem a respeito da tecnologia da iluminação aplicada à arquitetura e boas práticas da iluminação.

O manual The IES Lighting Handbook (IES, 2011) reúne no capítulo 18 - Iluminação Residencial, um conjunto de indicadores de iluminação para as diversas atividades exercidas nos ambientes residenciais. As recomendações de conforto visual consideram as iluminâncias associadas à faixa de idade dos usuários do ambiente, a uniformidade da iluminação e a refletância das superfícies internas (Tabela 1). Outros parâmetros como a temperatura de cor correlata, índice de reprodução de cor, os diversos efeitos luminotécnicos produzidos, suas aplicações e dispositivos de controle são apresentados para as diferentes atividades.

Semelhante ao manual IES (2011), os principais estudos realizados pelos membros pesquisadores da SSL estão reunidos nas publicações SLL Lighting Handbook (SSL, 2009) e na coletânea SLL Lighting Guides (SSL, 2013). O Guia LG 09, Lighting for Communal Residential Buildings (SLL, 2013), reúne um conjunto de orientações consideradas como boas práticas de iluminação em ambientes residenciais, podendo variar de um pequeno 
apartamento até os diferentes tipos de residências. A Tabela 2 a seguir sintetiza as principais recomendações para realização das atividades em ambientes residenciais.

\begin{tabular}{l} 
Tabela 1 - Principais recomendações de lluminâncias - IES (2011) \\
\hline Ambiente \\
\hline
\end{tabular}

\begin{tabular}{lccccccc}
\hline Jantar & & & & & & & \\
\hline Formal & 25 & 50 & 100 & 10 & 20 & 40 & $4: 1$ \\
\hline Informal & 50 & 100 & 200 & 20 & 40 & 80 & $4: 1$ \\
\hline Quartos & & & & & & & \\
\hline Mesas & 200 & 400 & 800 & 37,5 & 75 & 150 & \\
\hline Geral ( vestir) & 25 & 50 & 100 & 15 & 30 & 60 & $3: 1$ \\
\hline Leitura na cama & 100 & 200 & 400 & 50 & 100 & 200 & \\
\hline Assistir televisão & 15 & 30 & 60 & 7,5 & 15 & 30 & $3: 1$ \\
\hline Cozinha & & & & & & & \\
\hline Área café da manhã & 100 & 200 & 400 & 25 & 50 & 100 & $5: 1$ \\
\hline Armários & & & & 25 & 50 & 100 & $3: 1$ \\
\hline Cooktops & 150 & 300 & 600 & 25 & 50 & 100 & $2: 1$ \\
\hline Geral & 25 & 50 & 100 & 10 & 20 & 40 & $5: 1$ \\
\hline Preparo & 250 & 500 & 1000 & 37,5 & 75 & 150 & $2: 1$ \\
\hline Pias & 150 & 300 & 600 & 25 & 50 & 100 & $2: 1$ \\
\hline
\end{tabular}

Sendo: E = lluminâncias; U= Uniformidade. Fonte: IES (2011). Lighting for Residences, adaptado pelos autores.

\begin{tabular}{lccc}
\multicolumn{4}{l}{ Tabela 2 - Principais recomendações do Guide 9 - SSL (2013) } \\
\hline \multicolumn{1}{c}{ Ambiente } & $\begin{array}{c}\mathrm{E}_{\mathrm{M}} \\
\text { (lux) }\end{array}$ & $\begin{array}{c}\mathrm{T}_{\mathrm{cp}} \\
\text { (K) }\end{array}$ & $\mathbf{R}_{\mathbf{a}}$ \\
\hline Sanitários & & & \\
\hline Toilets & 100 & $>85$ \\
\hline Banheiro & 150 & $>85$ \\
\hline Quartos & & & - \\
\hline Geral & 100 & $2.700-3.000$ & $>85$ \\
\hline Bancada & Adicional de 150 & $>85$ \\
\hline Na cabeceira & 100 & $>85$ \\
\hline Salas & 100 & $>85$ \\
\hline Sala de estar & 100 & $>85$ \\
\hline Sala de tv & 50 & $>85$ \\
\hline Para leitura & Adicional de 200 & $>85$ \\
\hline Cozinha & 200 & $3.000-4.000$ & $>85$ \\
\hline Sala de jantar & 150 & 2.700 & $>85$ \\
\hline Fonte: Guide LG 09 (SL, 2013), adaptado pelos autores
\end{tabular}

Fonte: Guide LG 09 (SLL, 2013), adaptado pelos autores

Os dois manuais consideram, ainda, outras variáveis como a eficiência energética e a gestão da energia, com uso de sistemas de controle da luz.

Como nos manuais, as regulamentações também apresentam um conjunto de indicadores da iluminação, porém eles estão instituídos como valores mínimos de desempenho a serem alcançados pelas edificações. Muitos deles expressam as características e particularidades de cada país ou grupo de países onde a regulamentação se encontra em vigor. As principais regulamentações são:

\section{Associação Brasileira de Normas Técnicas - ABNT}

No Brasil, encontram-se vigentes duas normas que instituem indicadores de desempenho da iluminação artificial. A NBR 15575-1 - Norma de Desempenho das Edificações Habitacionais (ABNT, 2013a) e a NBR ISO/CIE 8995-1 - Iluminação de ambientes de trabalho. Parte 1: Interior (ABNT, 2013b) dedicada à tipologia não residencial.

A Norma de Desempenho das Edificações Habitacionais (ABNT, 2013a) estabelece no item 13 o desempenho da iluminação artificial das habitações brasileiras. Os diferentes ambientes dos edifícios habitacionais devem atender a níveis de iluminamento gerais médios (M). Os níveis intermediário (I) e superior (S) são sugestões para ambientes que buscam maior conforto dos usuários (Tabela 3).

Tabela 3 - Níveis de iluminamento geral para iluminação artificial das habitações brasileiras

\begin{tabular}{|c|c|c|c|}
\hline \multirow[t]{2}{*}{ Ambiente } & \multicolumn{3}{|c|}{$\begin{array}{l}\text { lluminamento geral para os } \\
\text { níveis de desempenho (lux) }\end{array}$} \\
\hline & $(\mathrm{M})$ & (l) & (S) \\
\hline Sala de estar & \multirow{4}{*}{$\geq 100$} & \multirow{4}{*}{$\geq 150$} & \multirow{4}{*}{$\geq 200$} \\
\hline Dormitório & & & \\
\hline Banheiro & & & \\
\hline Área de serviço Garagens & & & \\
\hline Copa/cozinha & $\geq 200$ & $\geq 300$ & $\geq 400$ \\
\hline $\begin{array}{l}\text { Corredor ou escada interna à } \\
\text { unidade }\end{array}$ & $\geq 100$ & $\geq 150$ & $\geq 200$ \\
\hline
\end{tabular}

Sendo: $(M)$ = Valores mínimos obrigatórios; $(\mathrm{I})$ = Níveis intermediários; $(\mathrm{S})$ = Níveis superior. Fonte: Anexo E da NBR 15575-1 (ABNT, 2013a), adaptado pelos autores

As iluminâncias instituídas na norma de Desempenho das Habitações (ABNT, 2013a) constam da extinta NBR 5413 (ABNT, 1992). A retomada desses indicadores demonstra a importância de parâmetros de desempenho de conforto visual em habitações, uma vez que a norma atual, a NBR ISO/CIE 8995-1 (ABNT, 2013b), não inclui a tipologia residencial.

\section{International Organization for Standardization - ISO}

A ISO é uma organização internacional que reúne associações ou instituições representativas da normalização de vários países, incluindo o Brasil (representado pela ABNT), para desenvolvimento de normas internacionais.

A ISO CIE 8995-1 (ABNT, 2013b) apresenta os requisitos para o planejamento da iluminação, atribuindo a cada tipo de ambiente uma iluminância média, um índice limite de ofuscamento $\left(\mathrm{UGR}_{\mathrm{L}}\right)$ e um índice de reprodução de cor mínimo $\left(\mathrm{R}_{\mathrm{a}}\right)$. Apesar de ser uma norma dedicada ao setor não residencial, algumas das atividades apresentadas são compatíveis com muitas das atividades residenciais (Tabela 4). 
Em conjunto com os requisitos do planejamento da iluminação, a norma considera a influência do entorno imediato à tarefa exercida, de modo a permitir uma distribuição balanceada da luz, e evitar mudanças drásticas de iluminância que afetam o conforto visual (Tabela 5).

Tabela 4 - Ambientes (áreas), tarefas, e atividades com a especificação de Iluminância, limitação de ofuscamento e qualidade da cor

\begin{tabular}{lccc}
\multicolumn{1}{c}{$\begin{array}{c}\text { Tipo de tarefa, ambiente } \\
\text { ou atividade }\end{array}$} & $\begin{array}{c}\mathbf{E}_{\mathbf{M}} \\
\text { (lux) }\end{array}$ & UGRL & $\mathbf{R}_{\mathbf{a}}$ \\
\hline Áreas gerais da edificação & 200 & 22 & 80 \\
\hline Sala de espera & 100 & 28 & 40 \\
\hline Áreas de circulação e corredores & 200 & 22 & 80 \\
\hline Refeitório / cantina & 100 & 22 & 80 \\
\hline Salas de descanso & 200 & 25 & 80 \\
\hline Vestiários/ banheiros/ toaletes & & & \\
\hline Lavanderias e limpeza a seco & 300 & 25 & 80 \\
\hline Lavagem e limpeza a seco & 300 & 25 & 80 \\
\hline Passar roupa & & & \\
\hline $\begin{array}{l}\text { Escritório } \\
\text { Escrever, teclar, ler e } \\
\text { processar dados }\end{array}$ & 500 & 19 & 80 \\
\hline $\begin{array}{l}\text { Restaurantes e hotéis } \\
\text { Cozinha }\end{array}$ & 200 & 22 & 80 \\
\hline $\begin{array}{l}\text { Restaurante, sala de jantar, } \\
\text { sala de eventos }\end{array}$ & & & \\
\hline
\end{tabular}

Fonte: NBR ISO/CIE 8995-1(ABNT, 2013b), adaptado pelos autores

\begin{tabular}{cc} 
Tabela 5 - lluminâncias mantidas na área do entorno imediato \\
\hline $\begin{array}{c}\text { Iluminância da tarefa } \\
\mathrm{E}_{M} \text { (lux) }\end{array}$ & $\begin{array}{c}\text { Numinância do entorno imediato } \mathrm{E}_{\mathrm{M}} \\
\text { (lux) }\end{array}$ \\
\hline$\geq 750$ & 500 \\
\hline 500 & 300 \\
\hline 300 & 200 \\
\hline$\leq 200$ & $\begin{array}{c}\text { Mesma lluminância da área de } \\
\text { trabalho }\end{array}$ \\
\hline
\end{tabular}

Fonte: NBR ISO CIE 8995-1(ABNT, 2013b), adaptado pelos autores

\section{European Committee for Standardization - CEN}

As normas utilizadas pela Comunidade Europeia são instituídas pelo CEN e válidas para todos os países integrantes. As questões relativas ao desempenho da iluminação estão descritas na EN 12464-1. Light and lighting - Lighting of work places - Part 1: Indoor work places (CEN, 2011).

A norma descrimina os ambientes interiores, tarefas ou atividades para o setor industrial, comercial e público. $\mathrm{O}$ setor residencial não é contemplado pela norma. Não obstante, a Tabela 6 a seguir demonstra atividades existentes nas áreas gerais de edifícios que podem servir como referência para a temática residencial.

Semelhante à ISO 8995-1 (ABNT, 2013b), a norma considera a influência do entorno imediato à tarefa exercida e traz recomendações da relação de Iluminâncias para realização da tarefa e o entorno imediato, com valores semelhantes à ISO CIE 8995-
1(ABNT, 2013b). Apresenta também parâmetros para a proteção contra o ofuscamento e outros requisitos.

\begin{tabular}{lcccc} 
Tabela 6 - Requisitos mínimos para a iluminação segundo a EN 12464-1 (2011) \\
\hline \multicolumn{1}{c}{ Tipo de área, tarefa ou atividade } & $\begin{array}{c}\text { Em } \\
\text { (lux) }\end{array}$ & UGR & $\mathbf{U}_{\mathbf{0}}$ & $\mathbf{R}_{\mathbf{a}}$ \\
& 200 & 22 & 0,4 & 80 \\
\hline Cantinas e copas & 100 & 22 & 0,4 & 80 \\
\hline Quarto de descanso & 200 & 25 & 0,4 & 80 \\
\hline Vestiários, banheiros e sanitários. & 500 & 22 & 0,6 & 80 \\
\hline Cozinha & - & - & - & 80 \\
\hline $\begin{array}{l}\text { Restaurante, sala de jantar, sala de } \\
\text { eventos. }\end{array}$ & 100 & 25 & 0,4 & 80 \\
\hline Corredores &
\end{tabular}

Fonte: EN 12464-1(CEN,2011), adaptado pelos autores. Ver requisitos específicos descritos na norma

\section{American Society of Heating, Refrigerating and Air- Conditioning Engineers - ASHRAE}

Nos Estados Unidos, os requisitos mínimos de eficiência energética em unidades habitacionais estão descritos na ASHRAE Standard 90.2 Energy-Efficient Design of Low-Rise Residential Buildings (ASHRAE, 2007). Essa norma traz recomendações de desempenho da envolvente da edificação, sistemas e equipamentos domésticos, dentre outros. Porém, não trata dos requisitos de desempenho da iluminação artificial.

Já a ASHRAE Standard 90.1 (ASHRAE, 2016). Energy Standard for Buildings Except Low-Rise Residential Buildings é mais abrangente, abordando as tipologias comerciais, públicas e de serviços. A sessão 9 (Lighting) da norma estabelece os requisitos mínimos de desempenho energético dos sistemas de iluminação artificial instalados nas edificações.

O indicador de eficiência da iluminação é a densidade de potência de iluminação (DPI) e pode ser obtido por dois métodos, o método da área do edifício - The Building Area Method (Tabela 7) e o método espaço por espaço The Space-by-Space Method (Tabela 8).

Tabela 7 - Densidade de potência da iluminação artificial - The Building Area Method

\begin{tabular}{lc}
\hline \multicolumn{1}{c}{ Tipo de área da edificação } & DPI $\left(\mathbf{W} / \mathrm{ft}^{2}\right)$ \\
\hline Dormitório & 0.61 \\
\hline Escritórios & 0.79 \\
\hline Jantar: Família & 0.78 \\
\hline Multifamiliar & 0.68
\end{tabular}

Fonte: ASHRAE (2016), adaptado pelos autores

Tabela 8 - DPI pelo método Space- by -Space Tipos espaço comum

Área de Refeições

Em uma instalação para deficientes visuais $\quad 2.00$

No jantar da família

0.71

Todas as outras áreas de jantar 0.63

Quarto de hóspedes $\quad 0.77$

Lavandaria / Área de lavar roupa 0.43

Dormitório - alojamento

0.54

Fonte: ASHRAE (2016), adaptado pelos autores 
O método da área considera o tipo da edificação e a área iluminada do andar. Já o método espaço por espaço é aplicado considerando cada ambiente da edificação separadamente, utilizando a relação de ambientes estipuladas na norma. Caso o ambiente apresente funções múltiplas, o ambiente será dividido em subespaços menores.

\section{Building Energy Efficiency Standards}

O estado americano da Califórnia, Estados Unidos, desenvolveu leis estaduais mais rigorosas para a questão da eficiência energética nas edificações e para a iluminação artificial em ambientes residenciais (CEC, 2015). O código da Califórnia, Building Energy Efficiency Standards for Residential and Nonresidential Buildings, no título 24, Parte 6 (CEC, 2017) apresenta os requisitos de desempenho a serem atendidos pela iluminação residencial.

O referido código determina o uso de sistemas de iluminação de alta eficiência atuando em conjunto com sensores e dispositivos de controle da luz. Os sistemas de iluminação considerados de alta eficiência devem atender aos requisitos que associam aspectos de conforto visual e eficiência luminosa (Tabela 9).

Tabela 9 - Principais requisitos de qualificação para alta eficiência das fontes de luz segundo o item JA8 do Código da Califórnia

\begin{tabular}{cc}
\hline Especificação & Exigência \\
\hline Eficiência inicial & $\geq 45$ lúmens/W \\
\hline Temperatura de Cor & $\leq 4.000 \mathrm{~K}$ \\
& $\leq 3.000 \mathrm{~K}$ \\
IRC & (A maioria das fontes) \\
\hline Vida útil & $\geq 90 \%$ \\
\hline Nível de dimerização & $\geq 15.000 \mathrm{~h}$ \\
\hline
\end{tabular}

Fonte: CLTC ( 2016), adaptado pelos autores

Os requisitos adicionais de qualificação estão detalhados no item JA8 da referida norma. Nesse item são definidas as particularidades das diferentes fontes de luz e luminárias, incluindo aquelas usadas para fins de iluminação decorativa e as luminárias em LED com fontes de luz integradas, que devem ser certificadas pela Comissão de Energia, cumprindo requisitos específicos.

\section{The Building Code of Australia - BCA}

$\mathrm{O}$ código de obras da Austrália, The Building Code of Australia - BCA (ABCB, 2016a, 2016b), contém as disposições técnicas para a concepção e construção de edifícios no país. A iluminação e eficiência energética são reguladas através de padrões mínimos de desempenho especificados na Seção J6.2 Artificial lighting.

O BCA considera as tipologias de edificações separadas em 10 classes. A tipologia residencial encontra-se contemplada nas classes 1, 2 e 4 (ABCB, 2016a, 2016b).
A eficiência energética dos sistemas de iluminação nos ambientes internos residenciais não deve exceder $5 \mathrm{~W} / \mathrm{m}^{2}$ nos ambientes da habitação e $4 \mathrm{~W} / \mathrm{m}^{2}$ nas áreas de varanda (ou similar) ligados à unidade de ocupação. $\mathrm{O}$ referido código permite a utilização de um fator de ajuste na DPI, caso o sistema de iluminação possua dispositivos de controle. Esse valor pode variar entre 0,5 e 0,95, a depender do ambiente, dispositivo de controle e o sistema de iluminação proposto.

As edificações contidas nas demais classes são as tipologias comerciais, de serviços ou públicas e devem atender a requisitos de DPI $_{L}$ como demonstrados na Tabela 10.

Tabela 10 - Densidade de potência máxima da iluminação segundo o BCA

\begin{tabular}{lc}
\multicolumn{1}{c}{ Ambiente } & $\begin{array}{c}\mathrm{DPl} \\
\left(\mathbf{W} / \mathrm{m}^{2}\right)\end{array}$ \\
\hline Áreas comuns, espaços e corredores em um prédio de classe 2 & 8 \\
\hline $\begin{array}{l}\text { Dormitório de um edifício de classe 3 utilizado com a função de } \\
\text { dormir apenas }\end{array}$ & 6 \\
\hline $\begin{array}{l}\text { Dormitório de um edifício de classe 3 com a função de dormir e } \\
\text { estudo }\end{array}$ & 9 \\
\hline Cozinha e área de preparação de alimentos & 8 \\
\hline $\begin{array}{l}\text { Escritório - iluminado artificialmente com nível de iluminamento } \\
\text { de 200 lux ou mais }\end{array}$ & 9 \\
\hline $\begin{array}{l}\text { Escritório - iluminado artificialmente com nível de iluminamento } \\
\text { inferior a 200 lux }\end{array}$ & 7 \\
\hline Fonte: ABCB (2016a), adaptado pelos autores &
\end{tabular}

A DPI $I_{L}$ para as atividades e ambientes não descritos no BCA (ABCB, 2016) podem ser estimadas através da iluminância pretendida para cada ambiente (Tabela 11).

Tabela 11 - Densidade de Potência máxima da iluminação pela iluminância pretendida

\begin{tabular}{cc}
$\begin{array}{c}\text { Iluminância pretendida } \\
\mathbf{E}(\mathbf{l u x})\end{array}$ & $\begin{array}{c}\mathbf{D P L _ { L }} \\
\left(\mathbf{W} / \mathbf{m}^{2}\right)\end{array}$ \\
\hline $\mathrm{E} \geq 80$ & 7,5 \\
\hline $80<\mathrm{E} \leq 160$ & 9,0 \\
\hline $160<\mathrm{E} \leq 240$ & 10 \\
\hline $240<\mathrm{E} \leq 320$ & 11 \\
\hline $320<\mathrm{E} \leq 400$ & 12 \\
\hline $400<\mathrm{E} \leq 480$ & 13 \\
\hline $480<\mathrm{E} \leq 540$ & 14 \\
\hline $540<\mathrm{E} \leq 620^{*}$ & 15 \\
\hline
\end{tabular}

Fonte: ABCB (2016a), adaptado pelos autores. *Para níveis de iluminamento superiores a 620 lux, a eficiência luminosa média da fonte de luz não deve ser inferior a 80 Lúmens/W

\section{Documento Básico - DB}

$\mathrm{Na}$ Espanha, os indicadores relativos ao desempenho da iluminação artificial concentram-se em dois principais regulamentos, a EN 12464-1 (CEN, 2011) e no código de obras do país, intitulado Documento Básico - DB, HE-3 Eficiencia Energética de las Instalaciones de Iluminación (MINISTÉRIO DE FOMENTO, 2013).

As recomendações apresentadas na sessão HE3 excluem a aplicação do código no interior de habitações, porém o 
documento possui indicadores para as atividades afins ao uso residencial (MINISTÉRIO DE FOMENTO, 2013).

Este documento se destaca por instituir o Valor da Eficiência Energética da Instalação de Iluminação (VEEI), valor que mede a eficiência energética da iluminação de uma área de atividade distinta, cuja unidade de medida é $\mathrm{W} / \mathrm{m}^{2}$ por cada 100 lux (Tabela 12). $\mathrm{Na}$ literatura brasileira esse indicador é denominado densidade de potência relativa $\left(\mathrm{DPI}_{\mathrm{R}}\right)$.

O Documento recomenda ainda a DPI dos sistemas de iluminação em função do uso do edifício. A Tabela 13 demonstra a DPI para atividades afins, como prédio público residencial e os meios de hospedagem.

Tabela 12 - Valor da Eficiência Energética da Instalação de lluminação (VEEI) de ambientes internos

\begin{tabular}{|c|c|}
\hline Áreas de atividade & $\begin{array}{c}\text { VEEI Limite } \\
\left(\mathrm{W} / \mathrm{m}^{2} \text { por cada } 100 \text { lux }\right)\end{array}$ \\
\hline Cozinhas & 4 \\
\hline Hotéis e restaurantes & 8 \\
\hline Quartos de hotel, pousada, etc. & 10 \\
\hline
\end{tabular}

Fonte: DB-HE3. (MINISTÉRIO DE FOMENTO, 2013), adaptado pelos autores

Tabela 13 - DPI máxima instalada das instalações de iluminação pelo uso do edifício

\begin{tabular}{lc}
\multicolumn{1}{c}{ Uso do edifício } & DPI máxima instalada $\left(\mathbf{W} / \mathbf{m}^{2}\right)$ \\
\hline Hospedagem & 15 \\
\hline Público residencial & 12 \\
\hline $\begin{array}{l}\text { Edifícios com nível de iluminação } \\
\text { superior a 600lux }\end{array}$ & 25 \\
\hline Fonte: DB-HE3 (MINISTÉRIO DE FOMENTO 2013), adaptado pelos autores
\end{tabular}

\section{Indicadores instituídos nas certificações}

As certificações brasileiras para edificações residenciais oriundas de iniciativas do Governo Federal são o Regulamento Técnico da Qualidade para o Nível de Eficiência Energética de Edifícios Residenciais - RTQ-R (ELETROBRAS; UFSC, 2014) e o Selo Casa Azul, emitido pela Caixa Econômica Federal (2010). As duas trazem um conjunto de recomendações no que se refere ao desempenho em edificações residenciais.

Os empreendedores ou usuários que desejem obter outras certificações de qualidade ambiental das edificações podem recorrer os selos internacionais. Das instituições internacionais, algumas já possuem referenciais considerando as características locais do Brasil como a Haute Qualité Environnementale - HQE, na versão brasileira denominada Alta Qualidade Ambiental AQUA (FUNDAÇÃO VANZOLINI, 2014) e a Leadership in Energy and Environmental Design LEED, na versão brasileira denominada GBC Brasil Casa e Condomínio, concedida pela Green Building Council Brasil (GBC BRASIL, 2014).

O RTQ-R apresenta as recomendações necessárias para a classificação da eficiência energética de unidades habitacionais autônomas (UH), e outros tipos como unifamiliares, multifamiliares. Os requisitos a serem atendidos pela iluminação artificial encontram-se no item bonificações como iniciativas que aumentam a eficiência da UH.

O desempenho da iluminação é determinado através de limites mínimos de eficiência luminosa. O melhor desempenho pode ser obtido quando os ambientes das UH possuírem todas as fontes de iluminação com eficiência luminosa superior a $75 \mathrm{~lm} / \mathrm{W}$ ou que as mesmas tenham obtido Selo PROCEL (ELETROBRAS; UFSC, 2014).

Diferente do RTQ-R, os Requisitos Técnicos da Qualidade para o Nível de Eficiência Energética de Edifícios Comerciais, de Serviços e Públicos - RTQ-C (ELETROBRAS; UFSC, 2015) determinam diretrizes para o dimensionamento da iluminação artificial utilizando o indicador $\mathrm{DPI}_{\mathrm{L}}$. Dois métodos podem ser utilizados para avaliar a eficiência energética do edifício, o método da área do edifício (Tabela 14) e o das atividades do edifício (Tabela 15).

O método da área da edificação avalia o sistema de iluminação de forma geral, aplicado quando a edificação possua no máximo três atividades principais ou quando as atividades ocupam mais de $30 \%$ da área do edifício. A Tabela 14 apresenta a aplicação do método.

Tabela 14 - DPI $L$ pelo método da área da edificação

\begin{tabular}{lcccc}
\hline Função da edificação & \multicolumn{4}{c}{ DPIL (W/m² $)$} \\
\hline & Nível A & Nível B & Nível C & Nível D \\
\hline Hotel & 10,8 & 12,4 & 14 & 15,7 \\
\hline Restaurante & 9,60 & 11,00 & 12,50 & 13,90 \\
\hline Hospedagem, dormitório & 6,60 & 7,60 & 8,60 & 9,60 \\
\hline Fonte: RTQ-C (ELETROBRAS; UFSC 2015$)$ & adaptado pelos autores
\end{tabular}

Fonte: RTQ-C (ELETROBRAS; UFSC, 2015), adaptado pelos autores

Tabela 15 - DPI L pelo Método das atividades do edifício

\begin{tabular}{|c|c|c|c|c|c|c|}
\hline \multirow[t]{2}{*}{ Ambiente / atividade } & \multicolumn{2}{|c|}{ Limite } & \multicolumn{4}{|c|}{$\mathrm{DPl}\left(\mathrm{W} / \mathrm{m}^{2}\right)$} \\
\hline & 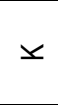 & $\begin{array}{l}\stackrel{\Upsilon}{\bigcup} \\
\text { ㄸ }\end{array}$ & $\frac{\longleftarrow}{\stackrel{\Xi}{ \pm}}$ & $\frac{\Phi}{\stackrel{\Phi}{\Sigma}}$ & $\frac{0}{\stackrel{0}{\perp}}$ & $\frac{\square}{\stackrel{D}{\perp}}$ \\
\hline Lavanderia & 1,2 & 4 & 6,5 & 7,8 & 8,1 & 10,4 \\
\hline Quartos Hotel & 0,8 & 6 & 7,5 & 9,0 & 10,5 & 13,0 \\
\hline Restaurante- hotel & 1,2 & 4 & 8,8 & 10,56 & 12,32 & 14,08 \\
\hline Espera - convivência & 1,2 & 4 & 6,0 & 7,2 & 8,40 & 9,60 \\
\hline
\end{tabular}

Onde: RCR: Room Cavity Ratio (adimensional); K: índice de ambiente (adimensional). Fonte: RTQ-C (ELETROBRAS; UFSC, 2015), adaptado pelos autores

\section{Análise dos indicadores}

A investigação demonstrou a existência de uma ampla diversidade de indicadores de desempenho da iluminação artificial. O Quadro 2 apresenta uma síntese dos documentos consultados e os principais indicadores encontrados, com marcadores que identificam a natureza do documento e a tipologia da edificação abordada. 
Os indicadores relativos ao conforto visual parecem ser suficientemente abordados pelas publicações examinadas. Como exemplo, a NBR ISO/CIE 8995-1 (ABNT, 2013b) juntamente com a EN 12464-1 (CEN,
2011) são normas que contemplam uma grande diversidade de países. As duas reúnem as principais recomendações em conforto visual para ambientes no setor não residencial.

Quadro 2 - Síntese dos principais indicadores presentes nas publicações consultadas

\begin{tabular}{|c|c|c|c|c|c|c|c|c|c|c|}
\hline \multicolumn{2}{|r|}{ 2-sintese vos pintis? } & \multicolumn{9}{|c|}{ Indicadores } \\
\hline \multirow{2}{*}{\multicolumn{2}{|c|}{ Pesquisa Dirigida }} & \multicolumn{5}{|c|}{ Conforto do usuário } & \multicolumn{4}{|c|}{ Eficiência } \\
\hline & & Ем (lux) & $U_{0}$ & UGRL & $\mathrm{Ra}_{\mathrm{a}}$ & $\mathrm{T}_{\text {сp }}(\mathrm{K})$ & $\begin{array}{c}\mathrm{DPI} \\
\left(\mathrm{W} / \mathrm{m}^{2}\right)\end{array}$ & $\begin{array}{c}\mathrm{DPI} \\
\left(\mathrm{W} / \mathrm{m}^{2}\right)\end{array}$ & $\begin{array}{c}\mathrm{DPI_{R }} \\
\left(\mathrm{W} / \mathrm{m}^{2}\right. \\
100 \text { lux }\end{array}$ & $\eta([\mathrm{m} / \mathrm{W})$ \\
\hline$\frac{\mathscr{s}}{\bar{\pi}}$ & The IES Lighting Handbook (IES, 2011). & 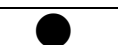 & O & & & 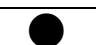 & & & & \\
\hline$\sum_{\substack{\mathrm{m} \\
\mathrm{m}}}$ & $\begin{array}{l}\text { Lighting for Communal Residential Buildings } \\
\text { (SLL, 2013). }\end{array}$ & O & & & & & & & & \\
\hline \multirow{7}{*}{ 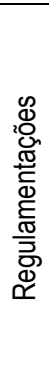 } & NBR 15575-1(ABNT, 2013a). & $\theta$ & & & & & & & & \\
\hline & NBR ISO/CIE 8995-1(ABNT, 2013b). & & & & & & & & & \\
\hline & EN 12464-1 (CEN, 2011). & & & 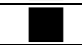 & & 1 & & & & \\
\hline & ASHRAE Standard 90.1 (ASHRAE, 2016). & & & & & & & & & \\
\hline & $\begin{array}{l}\text { Building Energy Efficiency Standards for } \\
\text { Residential Buildings (CEC, 2017). }\end{array}$ & & & & & & & & & \\
\hline & $\begin{array}{l}\text { The Building Code of Australia (ABCB, 2016a, } \\
\text { 2016b). }\end{array}$ & & & & & & & & & \\
\hline & $\begin{array}{l}\text { Código Técnico de la Edificación de España. } \\
\text { (MINISTÉRIO DE FOMENTO, 2013). }\end{array}$ & & & & & & & & & \\
\hline \multirow{3}{*}{ 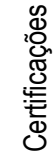 } & RTQ - C (ELETROBRAS; UFSC, 2015). & & & & & & $\square$ & & & \\
\hline & RTQ - R (ELETROBRAS; UFSC, 2014). & & & & & & & & & $\theta$ \\
\hline & $\begin{array}{l}\text { AQUA (FUNDAÇÃO VANZOLINI, 2014) e GBC } \\
\text { Brasil (2014) }\end{array}$ & $\theta$ & & & & & & & & $\theta$ \\
\hline
\end{tabular}

Fonte: Autores.

Os ambientes residenciais apresentam indicadores de conforto visual descritos predominantemente nos manuais de iluminação da IES (2011) e da SSL (2009, 2013), uma vez que dentre os documentos examinados não são encontradas regulamentações voltadas para a área residencial. A exceção ocorre com a Norma de Desempenho (ABNT, 2013a), onde são encontradas iluminâncias mínimas para ambientes residenciais. Já os indicadores de eficiência energética estão presentes predominantemente nas normas e códigos. Dentre os indicadores analisados, o indicador de densidade de potência limite $\left(\mathrm{DPI}_{\mathrm{L}}\right)$ apresenta-se como o mais difundido para o setor não residencial e o de eficiência luminosa o indicador mais aplicado ao setor residencial.

Porém, esta observação não se configura uma regra. $\mathrm{O}$ código de obras da Austrália (ABCB, 2016), por exemplo, estabelece o indicador de densidade de potência limite (DPI $)_{L}$ da iluminação para as tipologias residenciais do país. Apesar de ser generalista, por não detalhar as atividades exercidas nos ambientes internos, representa um avanço por instituir e abordar a questão residencial considerando os mesmos critérios das demais tipologias construtivas.

Dos indicadores de eficiência energética existentes nas regulamentações dedicadas aos ambientes residenciais, o código da Califórnia (CEC, 2017) se destaca das demais publicações por instituir um conjunto de indicadores que associam critérios de conforto visual e eficiência dos sistemas de iluminação residencial de forma integrada. Estes parâmetros podem ser observados ao estabelecer limites de eficiência luminosa, IRC e aparência de cor como principais critérios que definem a iluminação de alta eficiência.

Outro ponto forte do código da Califórnia (CEN, 2016b) está na aparência de cor recomendada para a iluminação de alta eficiência. A cor intermediária ou quente na iluminação, em que a temperatura de cor limite é de $4.000 \mathrm{~K}$, associada ao elevado índice de reprodução de cor trazem contribuições para manutenção do ciclo circadiano e seu efeitos sobre a supressão de melatonina noturna dos usuários em residências (FIGUEIRO, 2013; FIGUEIRO et al., 2014). Já o código espanhol (MINISTÉRIO DE FOMENTO, 2013) é pioneiro ao instituir a densidade de potência relativa $\left(\mathrm{DPI}_{\mathrm{R}}\right)$ como indicador para medir o desempenho da iluminação em regulamentações.

A DPI $_{R}$ possui como parâmetro de composição do indicador a iluminância mantida e a DPI do ambiente. Esta característica associa critérios de conforto visual e eficiência no ambiente simultaneamente em um mesmo 
indicador. $\mathrm{A} \mathrm{DPI} \mathrm{R}_{\mathrm{R}}$ é considerada um bom indicador para ambientes laborais, onde o critério mais importante é a uniformidade da luz no ambiente.

\section{Os Indicadores da iluminação em residências brasileiras}

A análise comparativa das semelhanças e diferenças mais significativas dentre os indicadores examinados confirma que os indicadores brasileiros são genéricos e apresentam pouco rigor se comparados com a maioria dos indicadores encontrados na literatura internacional.

Os indicadores de conforto visual das habitações brasileiras, expressos na Norma de Desempenho (ABNT, 2013b), consideram apenas iluminâncias mantidas em ambientes residenciais. Enquanto outros indicadores demandam valores mínimos para uniformidade, ofuscamento, aparência e reprodução de cor da luz (NBR ISO CIE 8995-1: 2013b); (EN 12464-1: 2011); (SLL, 2013). A Tabela 16 a seguir sintetiza os indicadores de desempenho brasileiros recomendados pelas normas com aplicação residencial.

Tabela 16 - Síntese dos principais indicadores recomendados para habitações no Brasil

\section{Normas/ Ambientes}

\begin{tabular}{cc}
\multicolumn{2}{c}{ Indicadores } \\
\hline Conforto visual & $\begin{array}{c}\text { Eficiência da } \\
\text { iluminação }\end{array}$ \\
\hline
\end{tabular}

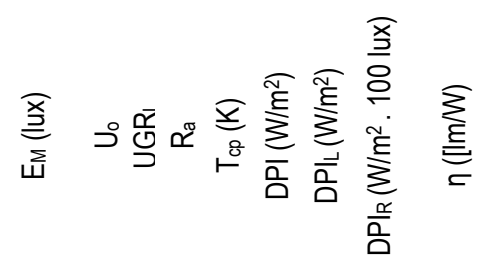

\begin{tabular}{|c|c|c|c|c|c|c|c|c|}
\hline $\begin{array}{l}\text { NBR 15575-1 } \\
\text { Salas, quartos, banheiro }\end{array}$ & $\geq 100$ & - & - & - & - & - & - & - \\
\hline $\begin{array}{l}\text { NBR 15575-1 } \\
\text { Cozinha }\end{array}$ & $\geq 200$ & - & - & - & - & - & - & - \\
\hline $\begin{array}{l}\text { RTQ - R } \\
\text { Todos os ambientes }\end{array}$ & - & - & - & - & - & - & - & $\geq 75$ \\
\hline
\end{tabular}

Fonte: NBR 15575-1(ABNT, 2013a); RTQ - R (ELETROBRAS; UFSC, 2014), adaptado pelos autores

O mesmo ocorre com o indicador de eficiência da iluminação. O RTQ-R (ELETROBRAS; UFSC, 2014) recomenda um valor mínimo de eficiência luminosa (75 $\mathrm{lm} / \mathrm{W}$ ) para as habitações que desejam desempenho energético. Já os indicadores da tipologia não residencial, como os instituídos no RTQ-C (ELETROBRAS; UFSC, 2015), são mais abrangentes, pois consideram os diversos usos da edificação, os ambientes internos e as atividades exercidas.

As certificações ambientais citadas neste trabalho, HQE/AQUA (FUNDAÇÃO VANZOLINI, 2014) e GBC Brasil (2014), por exemplo, possuem os documentos referenciais desenvolvidos para a realidade brasileira.
Essas certificações, ao considerar apenas os indicadores descritos nas regulamentações nacionais, limitam as possibilidades de inserção de novas tecnologias associadas a metas mais ousadas relativas ao desempenho energético da iluminação em ambientes residenciais.

A falta de um conjunto de indicadores que associem conforto visual e eficiência leva os projetistas brasileiros a recorrer a parâmetros de outras tipologias construtivas como as comerciais, de serviços e públicas, muitas vezes com critérios de desempenho diferentes do desejado para residências.

\section{Lacunas e Tendências}

A pesquisa documental indica importantes lacunas nos documentos examinados, à luz dos conhecimentos disponíveis nessa área de estudo.

Primeiramente, é importante frisar que as tipologias comercial e de serviços apresentam indicadores de desempenho amplamente descritos na literatura consultada, tanto na nacional quanto na internacional, concernentes ao conforto visual e relativo ao desempenho dos sistemas da iluminação no ambiente. Estes indicadores apresentam-se desagregados por tipo de tarefa ou atividade.

Apesar de existir nos documentos analisados uma separação notória entre os aspectos de conforto visual, por um lado, e desempenho energético, por outro, os indicadores apresentados para a tipologia não-residencial são conciliáveis e podem ter vasta aplicação em projetos com ênfase em desempenho energético. A necessidade de apresentá-los de maneira integrada é evidente, uma vez que esses indicadores estão diretamente relacionados.

As normas residenciais, em um dado momento, passaram a não ter atualizações com a mesma frequência dos demais setores. Torna-se evidente que os diversos parâmetros de projeto de iluminação para o setor residencial foram deixados a cargo do bom senso dos profissionais que atuam nesse segmento.

Uma vez que os indicadores instituídos nas normas brasileiras demonstram ser genéricos e pouco rigorosos, baseados apenas nos valores de iluminâncias mantidas, resta aos projetistas brasileiros buscarem referenciais de projetos em conforto visual utilizando os manuais, por exemplo, da IES (2011) ou o guia LG 09 (SSL, 2013).

No que concerne ao desempenho energético, como descrito anteriormente, os documentos analisados referentes à tipologia residencial concentram-se, em sua maioria, no desempenho energético dos equipamentos instalados, desconsiderando os impactos no conforto visual e no consumo de energia produzidos em função das diferentes configurações dos ambientes residenciais e 
nas atividades exercidas, verificando-se uma importante lacuna para o desenvolvimento de projetos de iluminação artificial.

O Estado da Califórnia - EUA (CEC, 2017) foi pioneiro ao conciliar questões relativas à qualidade da luz, saúde do usuário e desempenho energético, observando-se a inauguração de uma importante tendência de abordagem integrada. Porém, as recomendações para os sistemas em LED de alto desempenho (CLTC, 2016), não apresentam, ainda, uma boa aplicabilidade à realidade das residências brasileiras, seja devido ao alto custo, ou pela pouca oferta local desses equipamentos com IRC superior a 90, conforme pode ser constatado nos bancos de dados virtuais disponibilizados pelos fabricantes de lâmpadas e sistemas de iluminação em LED (DIAL GMBH, 2017).

Por fim, ampliando a abordagem dos aspectos trazidos pela experiência da Califórnia e verificando-se a tendência de abordar de forma integrada os aspectos fundamentais da iluminação artificial, propõe-se uma abordagem constituída por três pilares fundamentais da relação da luz nos ambientes residenciais: (I) os parâmetros necessários para realização de tarefas específicas, associadas à saúde e ao conforto visual dos usuários desses ambientes; (II) o desempenho lumínico dos equipamentos instalados; (III) o desempenho da iluminação considerando o efeito produzido no espaço habitado, no qual a estrutura composta se caracteriza pela forma da organização dos equipamentos nesses espaços. O indicador desejável deverá atender de forma integrada a esses três parâmetros.

Assim, defende-se que essas três abordagens componham a base teórica sobre a qual deverá se apoiar as novas normas. Vislumbram-se indicadores de desempenho mais rigorosos para o uso da luz artificial em residências, uma vez que saúde e desempenho estão intrinsecamente relacionados.

\section{Considerações finais}

A prática de projeto com ênfase em desempenho pressupõe a existência de um conjunto de indicadores que permitam a comparação do desempenho luminoso de diferentes soluções.

O presente trabalho demonstra a existência de grande número de indicadores da iluminação, instituídos nos manuais e regulamentos, para tipologias não residenciais. No entanto, faltam indicadores que melhor expressem o desempenho da iluminação artificial em ambientes residenciais, que considerem as particularidades do local, tipologias construtivas, tecnologias disponíveis e atividades exercidas nos ambientes.

A pesquisa documental sugere que o estado da arte caminha para o desenvolvimento de um conjunto de indicadores que associem conforto visual e eficiência energética, resultando na criação de novos paradigmas referentes aos projetos de iluminação artificial.

A etiquetagem da eficiência energética das edificações no Brasil pode ser considerada o marco inicial que institui o conceito de projeto residencial com ênfase em desempenho. Para tanto, torna-se imprescindível a utilização de indicadores que sirvam como ferramenta de projeto aplicada à arquitetura no contexto brasileiro, integrando as exigências de conforto visual com as demandas energéticas nas edificações residenciais.

Os aspectos acima apresentados ressaltam a importância da inclusão desses parâmetros nas normas vigentes, assim como, a difusão desse conhecimento na formação profissional, uma vez que a configuração dos ambientes habitados é determinada por estes projetistas.

\section{Agradecimentos}

À Coordenação de Aperfeiçoamento de Pessoal de Nível Superior - CAPES, pelo apoio a esta pesquisa através da concessão de bolsas de estudo nacional e no exterior.

\section{Referências}

ABCB - AUSTRALIAN BUILDING CODES BOARD. NCC 2016 Building Code of Australia. Volume One. Australia: ABCB, $2016 \mathrm{a}$.

ABCB - AUSTRALIAN BUILDING CODES BOARD. NCC 2016 Building Code of Australia. Volume Two. Australia: ABCB, $2016 b$.

ABNT - ASSOCIAÇÃO BRASILEIRA DE NORMAS TÉCNICAS. NBR 15575-1. Edificações Habitacionais. Desempenho Parte 1 : Requisitos gerais Prefácio. Rio de janeiro, 2013a. 60p. 
ABNT - ASSOCIAÇÃO BRASILEIRA DE NORMAS TÉCNICAS . NBR ISO/CIE 8995-1. Iluminação de ambientes de trabalho. Rio de janeiro, 2013b. 46 p.

ABNT - ASSOCIAÇÃO BRASILEIRA DE NORMAS TÉCNICAS. NBR 5413: Iluminância de interiores. Rio de janeiro, 1992. $13 \mathrm{p}$.

ASHRAE - AMERICAN SOCIETY OF HEATING, REFRIGERATING AND AIR-CONDITIONING ENGINEERS. ASHRAE Standard 90.1. Energy Standard for Buildings Except Low-rise Residential Buildings. Atlanta, 2016.

ASHRAE - AMERICAN SOCIETY OF HEATING, REFRIGERATING AND AIR-CONDITIONING ENGINEERS. ASHRAE Standard 90.2. Design of Low-Rise Residential Buildings. Atlanta, 2007.

BORGSTEIN, E.; LAMBERTS, R. Desenvolvimento de benchmarks nacionais de consumo energético de edificações em operação. São Paulo: CBCS, 2014. Disponível em: <www.cbcs.org.br/benchmarkingenergia〉, Acesso em: 5 Janeiro 2016.

CEC - CALIFORNIA ENERGY COMMISSION. California Building Energy Efficiency Standards for Residential and Nonresidential Buildings. California, 2015. Disponível em: < http://www.energy.ca.gov/2015publications/CEC-400-2015037/CEC-400-2015-037-CMF.pdf>. Acesso em: 10 Maio 2018.

CEC - CALIFORNIA ENERGY COMMISSION. Residential Lighting. In: 2016 Residential Compliance Manual. California, 2017. Disponível em: <http://www.energy.ca.gov/2015publications/CEC-400-2015-032/CEC-400-2015-032-CMF.pdf >. Acesso em: 10 Maio 2018.

CEN - EUROPEAN COMMITTEE FOR STANDARDIZATION. EN 12464-1. Light and lighting - Lighting of work places Part 1: Indoor work places. UK, 2011.

CLTC - CALIFORNIA LIGHTING TECHNOLOGY CENTER . RESIDENTIAL LIGHTING: WHAT'S NEW IN THE 2016 TITLE 24, PART 6 CODE? Publications. Guides. 02.08.2016. Disponível em: < https://cltc.ucdavis.edu/publication/2016-title-24code-changes-residential >. Acesso em: 05 Maio 2016.

CONSELHO BRASILEIRO DE CONSTRUÇÃO SUSTENTÁVEL. Benchmarking e etiquetagem energética "em uso" Visão Brasileira. São Paulo: CBCS, 2013. Disponível em: < http://www.cbcs.org.br/benchmarkingenergia >. Acesso em: 10 Maio 2014.

DIAL GMBH. LUMsearch. Easy search for luminaires online. DIAL, Software. Disponível em:

<https://www.dial.de/en/lumsearch/>. Acesso em: 01 Julho 2017.

EEA - EUROPEAN ENVIRONMENT AGENCY. EEA core set of indicators. EEA Technical report No 1/2005. Copenhagen: EEA, 2005. Disponível em: < https://www.eea.europa.eu/publications/technical_report_2005_1>. Acesso em: 10 Maio 2014.

ELETROBRAS; UFSC. Manual de aplicação do RTQ-C. 4.1. Versão 3. Brasilia: PROCEL, 2015. Disponivel em: 〈http://www.pbeedifica.com.br/sites/default/files/projetos/etiquetagem/comercial/downloads/manual_rtqc2016.pdf >. Acesso em: 10 Agosto 2017.

ELETROBRAS; UFSC. Manual para Aplicação do RTQ-R. 4.2. Brasilia: PROCEL, 2014. Disponível em: < http://www.pbeedifica.com.br/sites/default/files/projetos/etiquetagem/residencial/downloads/Manual_de_aplica\%C3\%A7\%C3\%A3 o_do_\%20RTQ-R-v01.pdf>. Acesso em 10 Agosto 2017.

FIGUEIRO, M. G.; PLITNICK, B.; REA, M. S. The effects of chronotype, sleep schedule and light/dark pattern exposures on circadian phase. Sleep Medicine, v. 15, n. 12, p. 1554-1564, 2014. doi:https://doi.org/10.1016/j.sleep.2014.07.009

FIGUEIRO, M. G. Why field measurements of circadian light exposure are important. Lighting Research \& Technology, v. 45, n. 1, p. 6-6, 2013. doi:http://dx.doi.org/10.1177/1477153512473709.

FUNDAÇÃO VANZOLINI. AQUA - HQE. Referencial de Avaliação da Qualidade Ambiental de Edifícios Residenciais em Construção. São Paulo: F. Vanzolini, 2014.

GBC BRASIL - GREEN BUILDING COUNCIL BRASIL. Referencial GBC Brasil Casa. São Paulo: GBC, 2014.

GRONDZIK, W. T.; KWOK, A. G. O processo de projeto. In: Manual de Arquitetura Ecologica. Porto Alegre: Bookman, 2013. 
IES - ILLUMINATING ENGINEERING SOCIETY. The Lighting Handbook - 10th Edition. New York: IES, 2011.

MINISTÉRIO DE FOMENTO. Código Técnico de la Edificación de España. Documento Básico DB-HE. Ahorro de Energía. BOLETÍN OFICIAL DEL ESTADO, p. 67137 - 67209. Madrid, 2013.

SSL - THE SOCIETY OF LIGHT \& LIGHTING. Lighting Guide 9: Lighting for Communal Residential Buildings. London: Chartered Institution of Building Services Engineers, 2013. 70p.

SSL - THE SOCIETY OF LIGHT \& LIGHTING. The SLL Lighting Handbook. London: Chartered Institution of Building Services Engineers, 2009. 328p.

\section{${ }^{1}$ Júlia Silva de Moraes}

Arquiteta. Mestre. Endereço Postal: Grupo de Estudos em Conforto Ambiental - GECA. Av. Lourival Melo Mota, s/n, Tabuleiro do Martins, Maceió, Alagoas, Brasil, 57072-900.

\section{${ }^{2}$ Adrián Muros Alcojor}

Arquiteto. Doutor. Endereço Postal: Taller de Estudios Lumínicos - TEL. Av. Diagonal, 649. Barcelona, Cataluña, España, 08028.

\section{${ }^{3}$ Leonardo Salazar Bittencourt}

Arquiteto. Doutor. Endereço Postal: Grupo de Estudos em Conforto Ambiental - GECA. Av. Lourival Melo Mota, s/n, Tabuleiro do Martins, Maceió, Alagoas, Brasil, 57072-900. 